\title{
A new thickness-based accelerated aging test methodology for resin materials: Theory and preliminary experimental study
}

\author{
Yanlei Wang ${ }^{a}$, Xue Zhang ${ }^{\mathrm{a}, *}$, Gaochuang Cai ${ }^{\mathrm{b}}$, Baolin Wan ${ }^{\mathrm{c}}$, Danièle Waldmann ${ }^{\mathrm{b}}$, Yuan $\mathrm{Qu}^{\mathrm{a}}$ \\ a State Key Laboratory of Coastal and Offshore Engineering, School of Civil Engineering, Dalian University of Technology, Dalian 116024, PR China \\ ${ }^{\mathrm{b}}$ Laboratory of Solid Structures, University of Luxembourg, Luxembourg L1359, Luxembourg \\ ' Department of Civil, Construction and Environmental Engineering, Marquette University, Milwaukee, WI 53201, USA
}

\section{H I G H L I G H T S}

- Thickness-based accelerated aging test method (ThAM) is firstly proposed for resin materials.

- Acceleration factors are theoretically deduced for water absorption and tensile behaviours of resin materials.

- Effects of specimen thickness on water absorption and tensile strength retention of resin materials are studied.

- The proposed ThAM ensures stable accelerated efficiency without changing the degradation mechanism of resin materials.

\section{A R T I C L E I N F O}

\section{Article history:}

Received 2 March 2018

Received in revised form 23 July 2018

Accepted 30 July 2018

\section{Keywords:}

Long-term durability

Water absorption

Tensile strength

Accelerated test methodology

Composite materials

\begin{abstract}
A B S T R A C T
This paper proposes a novel accelerated test method based on the thickness of resin materials. This method is to overcome the adverse influence of high temperature on the reliability of experimental results of the accelerated tests widely adopted in the current practice. To verify the proposed thickness-based accelerated method (ThAM), an experimental investigation was conducted focusing on the water absorption and tensile properties of epoxy resin. The results suggest that the existing temperature-based accelerated method (TAM) cannot be applied when the test temperature is high as in this case the degradation mechanism of materials is probably changed. The acceleration factor of TAM is greatly dependent on the type of test solution, which further limits the application in the accelerated test. Compared with TAM, the new method is much easier to apply, and more stable and reasonable to accelerate the aging test of epoxy resin.
\end{abstract}

(c) 2018 Elsevier Ltd. All rights reserved.

\section{Introduction}

In recent decades the repair and rehabilitation of existing structures have received increasing concerns [1-6]. Due to its convenience in construction, epoxy resin is widely used as an adhesive to bond external reinforcements (e.g. fibre reinforced polymer (FRP) sheets) to damaged structures. During the long service life of strengthened structures, the resin adhesives are generally exposed to aggressive environment such as wet-dry cycling and chloride ion attacks from de-icing salt [7-9]. The mechanical properties of hardened resin adhesive may be deteriorated after a longterm exposure to severe environments, which further affect the reliability of the strengthened structures. To confirm the longterm strengthening performance of the resin materials, the main challenge is how to accurately predict by quantification its long-

\footnotetext{
* Corresponding author.

E-mail address: xuezhang@dlut.edu.cn (X. Zhang).
}

term properties under such aggressive environment [10]. In the hygrothermal environment, water is easy to diffuse into the resin matrix, leading to the degradation of the adhesive. Therefore, many experimental studies have been conducted to reveal the impact of hygrothermal aging action on the properties of epoxy resin adhesive [11].

The water absorption of resin was tested for different durations under various natural environments, and its effect on the mechanical behaviour of resin was discussed [12-21]. These on-site aging tests can provide reliable results since that real exposed environment and degradation mechanism of resins can be ensured. One limitation of these studies is that, however, the duration of the natural aging tests varied from 36 to 1290 days which are all significantly shorter than their real service life. Thus, it is insufficient to evaluate the long-term durability of epoxy adhesives based on the current natural exposure results.

As a long-term on-site aging test of materials generally takes much time, accelerated aging testing methodologies are usually 
applied to obtain durability results of materials within a reasonable time [22-26]. In these methods, the test material was usually tested at a high aging rate by accelerating one of the main affecting factors of environment, and then the short-term properties of the material obtained from the aging test were transformed into its long-term properties via certain mathematical models. It is well known that the long-term degradation of epoxy resins mainly depends on moisture diffusion and chemical reactions when subjected to hygrothermal environment, and both factors can be accelerated by raising temperatures [27]. Therefore, the environmental temperature of the aging tests can be designed as one of the acceleration factors when the degradation mechanism of materials remains unchanged under different test temperatures. Since the development of the Arrhenius law [27], many tests based on the temperature-based accelerated method (TAM) have been widely adopted to evaluate the long-term durability of materials. Miyano et al. [22] and Miyano and Nakada [25] proposed a methodology to predict the long-term flexural fatigue life of carbon fibre reinforced polymer (CFRP) laminates used in marine environment. In their studies, elevated temperature states were used to accelerate the mechanical degradation, which occurred under loads over a long period of time at lower temperature. The fatigue strengths of various kinds of FRP, and structures under various types of loading situations were then predicted. Based on the short-term test data, Chen et al. [23] developed a detailed procedure to predict the long-term tensile behaviour of carbon fibre reinforced polymer (GFRP) bars in simulated concrete pore solutions. A modified Arrhenius analysis was included in the procedure to evaluate the validity of accelerated aging tests before the prediction was made. With the similar procedure, Chen et al. [24] predicted the longterm behaviours, including tensile strength, inter-laminar shear strength and bond strength, of both CFRP and GFRP bars under different environments. The results showed that elevated temperature accelerated the degradation of both bare FRP and FRPconcrete specimens. Recently, Wang et al. [26] proposed a refined prediction method for the long-term performance of basalt fibre reinforced polymer (BFRP) bars considering the effects of service year, concrete-wrap, environmental humidity and seasonal temperature fluctuations. According to available accelerated aging tests data, the reduction factors for the tensile strength of BFRP reinforcements in typical environments were predicted.

Although the TAM is effective for most of cases, it is still limited in practical application for the two following reasons [28]. First, the highest test temperature is generally lower than $60^{\circ} \mathrm{C}$ to ensure that the degradation of the test materials is similar to the actual situation, which results in a relative low accelerating efficiency for the tests. The existing investigations have demonstrated that, secondly, the degradation mechanism of resin matrix structure could be changed even at a moderate higher test temperature [28]. In this case, the elevated temperature would not only increase the rate of degradation but also influence the degradation mechanism, leading to a greater degradation compared to the case that the degradation mechanism is not changed. In other words, the deterioration of the properties of the resin materials may be overestimated through such accelerated aging tests. The aforementioned shortcomings are caused by that the test temperature is adopted as the acceleration factor in the TAM. Therefore, it is feasible to overcome these shortcomings by choosing other test variables instead of temperature to better accelerate aging tests of resin materials.

This paper aims to develop a new thickness-based accelerated test method (ThAM) as an alternative for accelerating aging tests of resin materials. By considering the water diffusion process in specimens with different thicknesses, the acceleration factors for both the mass gain and the tensile strength retention of the resin are theoretically deduced. To verify the new method, the water absorption and tensile strength of epoxy resin are then experimentally studied for a test duration of 180 days through TAM and ThAM investigations. The accelerating effect of aging tests of the resin materials based on the two different test methods are discussed in detail.

\section{Accelerated method for durability test}

\subsection{Temperature-based accelerated method}

The temperature-based accelerated method (TAM) is widely adopted to study the long-term durability of various materials. Arrhenius law [29] suggests that the relationship between the degradation rate $D$ and the Kelvin temperature $T$ can be expressed as Eq. (1),

$D=A \exp \left(-\frac{E_{a}}{R T}\right)$

where $A$ is a constant of the degradation process of the material, $E_{a}$ is the activation energy, and $R$ is the universal gas constant equalling to $8.314 \mathrm{~J} /(\mathrm{mol} \cdot \mathrm{K})$, respectively. Within the same test solution, an acceleration factor $\left(A F_{T}\right)$ of the accelerated tests at two different temperatures is defined as the ratio between the two required test durations to reach a given degradation level, which can be calculated by Eq. (2).

$A F_{T}=\frac{t_{1}}{t_{2}}=\frac{c / D_{1}}{c / D_{2}}=\frac{D_{2}}{D_{1}}=\frac{\exp \left(\frac{-E_{a}}{R T_{2}}\right)}{\exp \left(\frac{-E_{a}}{R T_{1}}\right)}=\exp \left[\frac{E_{a}}{R}\left(\frac{1}{T_{2}}-\frac{1}{T_{1}}\right)\right]$

where $t_{1}$ and $t_{2}$ are the required test durations to reach a given degradation level $c$ at temperatures $T_{1}$ and $T_{2}$, respectively. As a result, the test result obtained at a higher temperature can be used to predict the long-term behaviour of the material exposed to a lower temperature using Eq. (2) when the activation energy $E_{a}$ of the test solution is given. According to Eq. (2), the degradation mechanism should not be changed during the test requiring that the test temperature cannot exceed $60^{\circ} \mathrm{C}$. Moreover, the factor $A F_{T}$ depends on the test temperature and the activation energy of the test solution. In other words, when the test temperatures are same, a more significant acceleration effect can be ensured by using a solution with smaller activation energy. Therefore, the acceleration effect of the TAM may be limited when the solution with high activation energy is adopted in the test.

\subsection{Proposal of thickness-based accelerated method}

Previous experimental studies on FRP rods illustrate that the degradation rate of FRP materials is influenced by the diameter of the specimens [30]. Therefore, it is possible to explore a new accelerated methodology based on the thickness of the specimens. Similar efforts had been made by Dorkenoo and Pfromm [31] to investigate the effect of sample thickness on physical aging process of amorphous glassy polymer films. They established a thicknessdependent aging model to relate the gas permeability decay and sample thickness. This model, however, cannot be applied to design the accelerated hygrothermal aging test for resin materials because of the different degradation mechanism. In this section, therefore, the thickness-based accelerated method is theoretically proposed for the hygrothermal aging test of resin materials.

\subsubsection{Acceleration effect for long-term water absorption evaluation}

According to Bao et al. [32], the moisture uptake of most kinds of epoxy resin follows the relationship shown in Fig. 1. This indicates that the trend of water absorption of the epoxy resin is divided into two stages (i.e., Stages I and II) by a critical point at 


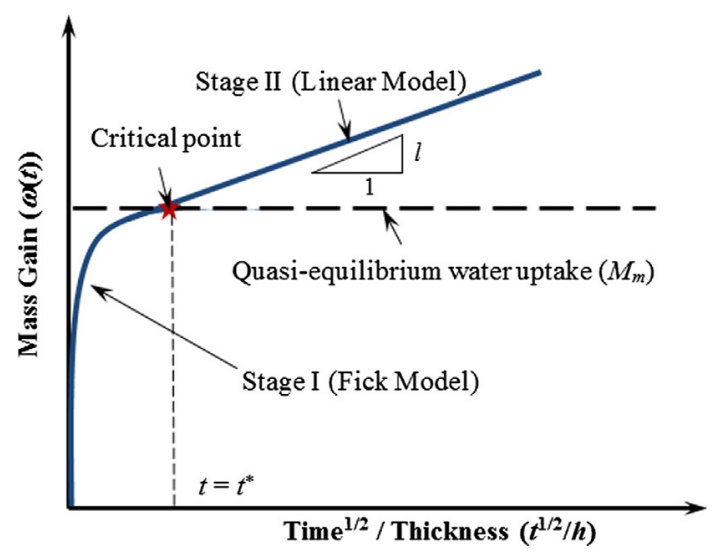

Fig. 1. Typical model for the water uptake of epoxy resin [32].

the aging time $t=t^{*}$. Before the critical point, the mass gain due to the water absorption $\omega(t)_{\mathrm{I}}$ increases gradually with aging time and finally reaches a quasi-equilibrium mass gain $\omega_{\mathrm{m}}$. In the stage I, it is assumed that the diffusion of resin material is determined by the concentration gradient of the test solution complying with the classic Fick's law, as shown in Eq. (3) [32].

$\omega(t)_{I}=\omega_{m}\left\{1-\exp \left[-7.3\left(\frac{D t}{h^{2}}\right)^{0.75}\right]\right\}$

where $\omega(t)_{\text {I }}$ is the mass gain at the first stage when the test time is $t, \omega_{m}$ is the quasi-equilibrium mass gain, and $h$ is the thickness of the specimen.

In the stage I, if the resin specimens with two thicknesses, i.e., $h_{1}$ and $h_{2}$, are tested to reach the same mass gain after aging time $t_{1}$ and $t_{2}$, a balanced equation can be obtained as:

$$
\begin{aligned}
\omega\left(t_{1}\right)_{I} & =\omega_{m}\left\{1-\exp \left[-7.3\left(\frac{D t_{1}}{h_{1}^{2}}\right)^{0.75}\right]\right\}=\omega\left(t_{2}\right)_{I} \\
& =\omega_{m}\left\{1-\exp \left[-7.3\left(\frac{D t_{2}}{h_{2}^{2}}\right)^{0.75}\right]\right\}
\end{aligned}
$$

Experimental results [32] show that the parameters of $\omega_{m}$ and $D$ in Eq. (4) are generally constant for a given resin and aging solution. This means that the mass gain changed by time is determined by the specimen thickness $h$. Therefore, the Eq. (4) is expressed as:

$t_{1} / t_{2}=\left(h_{1} / h_{2}\right)^{2}$

Based on Eq. (5), the aging times $\left(t_{1}^{*}\right.$ and $\left.t_{2}^{*}\right)$ corresponding to the critical points for the two specimens are also dependent on their thickness, i.e.,

$t_{1}^{*} / t_{2}^{*}=\left(h_{1} / h_{2}\right)^{2}$

On the other hand, the mass gain of non-Fickian material increases linearly with the aging time after it exceeds its quasiequilibrium level, as shown in Fig. 1. In the stage II, a linear relationship between the mass gain $\omega(t)_{\text {II }}$ and the aging time increment $\Delta t$ can be established as Eq. (7).

$\omega(t)_{\mathrm{II}}=\omega_{m}+l \cdot \frac{\sqrt{\Delta t}}{h}$

where $l$ is the slope of the development of mass gain shown in Fig. 1.

Similar to the stage I, if the resin specimens with two thicknesses $\left(h_{1}\right.$ and $\left.h_{2}\right)$ are tested with incremental aging times $\left(\Delta t_{1}\right.$ and $\Delta t_{2}$ ) to achieve a given mass gain, a balanced equation can also be established in the stage, which is given as:
$\omega\left(t_{1}\right)_{\mathrm{II}}=\omega_{m}+l \cdot \frac{\sqrt{\Delta t_{1}}}{h_{1}}=\omega\left(t_{2}\right)_{\mathrm{II}}=\omega_{m}+l \cdot \frac{\sqrt{\Delta t_{2}}}{h_{2}}$

Since the parameters $\omega_{m}$ and $l$ are both irrelevant to the specimen thickness $h$ [32], Eq. (8) is re-written as:

$\Delta t_{1} / \Delta t_{2}=\left(h_{1} / h_{2}\right)^{2}$

The acceleration factor of the specimens with different thickness subjected to an accelerated corrosion environment during total test period including both stages I and II can be expressed as,

$A F_{H}=\frac{t_{1}}{t_{2}}=\frac{t_{1}^{*}+\Delta t_{1}}{t_{2}^{*}+\Delta t_{2}}=\left(\frac{h_{1}}{h_{2}}\right)^{2}$

As a result, the acceleration factor $A F_{H}$ of mass gain due to water absorption based on the proposed accelerated test method can be calculated with Eqs. (5) and (10) for the Fickian and non-Fickian resins, respectively. It is shown that, for the specimens with different thicknesses, the ratio of required aging times to reach a given mass gain is inversely proportional to the square of their thickness ratio. Therefore, it is feasible to accelerate the aging test by reducing the thickness of the specimen.

\subsubsection{Acceleration effect for long-term tensile strength evaluation}

The nominal tensile strength $\sigma$, which is defined as Eq. (11), is used to evaluate the axial mechanical behaviour of the epoxy resin.

$\sigma=P / A$

where $P$ is the measured tensile load, $A$ is the cross-sectional area of the specimen.

The resin matrix is gradually eroded by the permeation of the environmental medium through the thickness of the specimen, which finally results in a degradation of the tensile strength of the materials. Fig. 2 shows the effect of corrosion on the specimen via single- or double-side permeation. When the total corrosion depth of the specimen reaches $x$, the time-dependent nominal tensile strength $\sigma(t)$ can be defined as,

$\sigma(t)=\frac{\sigma_{0} \cdot\left(A-A_{e}\right)+\sigma_{e} \cdot A_{e}}{A}=\frac{\sigma_{0} \cdot b \cdot(h-x)+\sigma_{e} \cdot b \cdot x}{b \cdot h}$

where $\sigma_{0}$ and $\sigma_{e}$ denote the initial and residual tensile strengths of the resin, respectively; $A_{e}$ is the area of eroded region; and $b$ and $h$ are the width and thickness of the specimen, respectively.

According to previous researches [33,34], for a layer-forming type corrosion shown in Fig. 2, the corrosion depth of the test resin, $x$, can be expressed as a function of the aging time, $t$, and given by, $x=\alpha \sqrt{t}$

where $\alpha$ is a constant of the materials which is independent on $h$.

Many studies $[1,11,14,16,18,19]$ have indicated that the actual residual tensile strength $\sigma_{e}$ of the resin is irrelevant to the specimen thickness and remains nearly constant after an long time duration. Substituting Eq. (13) into Eq. (12), the nominal tensile strength of the resin is given by:

$\sigma(t)=\sigma_{0}-\left(\sigma_{0}-\sigma_{e}\right) \frac{\alpha \sqrt{t}}{h}$

If the resin specimens with two thicknesses $\left(h_{1}\right.$ and $\left.h_{2}\right)$ are tested to achieve a given nominal tensile strength after duration time $t_{1}$ and $t_{2}$, a balanced equation can be obtained as,

$\sigma\left(t_{1}\right)=\sigma_{0}-\left(\sigma_{0}-\sigma_{e}\right) \frac{\alpha \sqrt{t_{1}}}{h_{1}}=\sigma\left(t_{2}\right)=\sigma_{0}-\left(\sigma_{0}-\sigma_{e}\right) \frac{\alpha \sqrt{t_{2}}}{h_{2}}$

According to Eq. (15), Eq. (5) can also be deduced to obtain the acceleration factor for the nominal tensile strength of resin materials. It should be noted that Eq. (5) is valid only when the total corrosion depth does not reach the thickness of specimen. Combining 


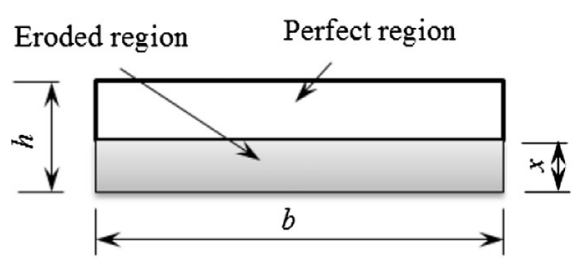

(a)

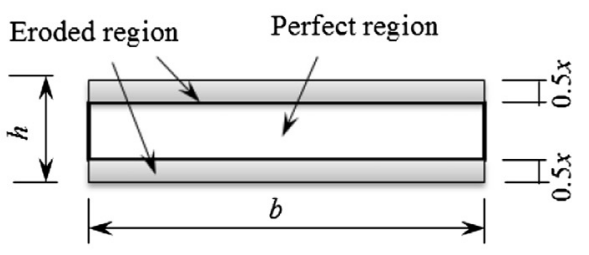

(b)

Fig. 2. Schematic of the specimen sections for tensile strength test: (a) single-side corrosion; (b) double-side corrosion.

with Eq. (13), this also suggests that Eq. (5) can be used to accelerate the test results when the test duration satisfies the condition of $t<(h / \alpha)^{2}$. Hojo et al. [34] reported that the value of the factor $\alpha$ is around $5 \times 10^{-3} \mathrm{~mm} / \mathrm{h}$, thus the effective duration of ThAM can be approximately estimated for a given specimen thickness. For example, if the thickness $h$ is set to $1 \mathrm{~mm}$, the effective aging time is about 40,000 h, i.e., 4.56 years. In other words, when the thickness of specimen is $1 \mathrm{~mm}$ in the aging test, the ThAM can be applied to evaluate the long-term tensile strength (up to 4.56 years) of the material.

According to the above discussion, it can be concluded that the proposed ThAM has two advantages compared with TAM: (1) a more significant acceleration effect could be obtained with the ThAM because the $A F_{H}$ is related to the second power of the specimen thickness; and (2) ThAM can be applied more easily and widely because the $A F_{H}$ is independent on the temperature and the activation energy of the test solution.

\section{Experimental program}

\subsection{Material and accelerated methodology}

To verify the proposed ThAM, an experimental program was designed to test the long-term water absorption and tensile strength of epoxy resin. A two-component commercial epoxy resin (JGN-T, produced by Kaihua Cooperation) was adopted in this study. The mixture ratio of the two components of the epoxy resin, i.e., the thixotropic epoxy adhesive and the resin binder, was 3:1 by weight. Polyethylene moulds were used to produce the resin specimens. The specimens were cured for $24 \mathrm{~h}$ at room temperature before removal from the moulds. According to the manufacturer, tensile strength, tensile modulus and elongation of the resin are $40 \mathrm{MPa}, 2.5 \mathrm{GPa}$, and $1.80 \%$, respectively.

The specimens were immersed in two types of liquids up to 180 days, i.e., distilled water and alkaline solutions. The maximum duration of 180-day is adopted to ensure that all specimens with different thicknesses can reach a quasi-equilibrium mass gain during the test. The alkaline solution $(\mathrm{PH}=13$ ) was prepared according to ACI 440.3R-04 Codes [35]. In the current study, TAM and ThAM were compared respectively in all specimens divided into two groups (i.e., Group A and B). The specific accelerating variables used in the two groups are listed in Table 1.

\subsection{Water absorption test}

The water absorption of the epoxy resin was evaluated by immersing the samples in distilled water or alkaline solution and

Table 1

Variable and constant parameters adopted in the test.

\begin{tabular}{llll}
\hline Group & Accelerated method & Test parameters & \\
\cline { 3 - 4 } & & Variable & Constant \\
\hline A & TAM & $T=23^{\circ} \mathrm{C}, 40^{\circ} \mathrm{C}, 60^{\circ} \mathrm{C}$ & $h=2 \mathrm{~mm}$ \\
B & ThAM & $h=1 \mathrm{~mm}, 2 \mathrm{~mm}, 4 \mathrm{~mm}$ & $T=60^{\circ} \mathrm{C}$ \\
\hline
\end{tabular}

then measuring their weight gain at specific periods. According to ASTM D570 [36], the dimension of the specimen was $60 \mathrm{~mm}$ $\times 60 \mathrm{~mm} \times h \mathrm{~mm}$, where $h$ was the thickness of the specimen. As listed in Table 1, the thickness $h$ of specimens in Group A was fixed as $2 \mathrm{~mm}$, while $h$ was set as three values in Group B because it was the main variable for ThAM.

Before testing, each cured specimen was dried in a drying oven at $60{ }^{\circ} \mathrm{C}$ for $48 \mathrm{~h}$ to remove its internal moisture, cooled down to the room temperature and then weighed to obtain its initial mass $M_{0}$. The specimen was then immersed in distilled water or alkaline solution for the aging test. After a given test duration, the specimen was surface-dried and weighed to get its aged mass $M_{t}$. The mass gain due to the water absorption of materials, $\omega$, can be expressed as Eq. (16). For the specimens in Groups A and B, the water absorption of the materials was tested at the same test duration shown in Table 2. For each given aging time, ten specimens were tested and the average values of all measured results were adopted in the following analyses.

$\omega=\frac{M_{t}-M_{0}}{M_{0}} \times 100$

\subsection{Tensile strength test}

In addition to the water absorption property, the tensile strengths of the epoxy resin specimens were also tested after the materials were subjected to a hydrothermal aging process. Dogbone-shaped resin specimens were prepared for uniaxial tensile strength tests according to ASTM D638 [37]. The ultimate tensile capacities of the specimens were tested after a given duration expressed as the nominal tensile strengths of the resin material which were calculated by Eq. (11). The test durations adopted in the studies are listed in Table 2. For each given aging time, five specimens were tested. The average values of the experimental results were adopted in the following analyses.

\section{Experimental results}

\subsection{Water absorption test}

For the specimens in Group A, the absorption test results for distilled water and alkaline solution are shown in Fig. 3(a) and (b), respectively. The mass gain $\omega$ was analysed against the square root of the aging time (in seconds) for different temperatures. Fig. 3 shows that the relationship between the mass gain and aging time is composed of two stages regardless of the immersing solution,

Table 2

Exposure time adopted in the test

\begin{tabular}{ll}
\hline Test & Exposure time adopted in the test \\
\hline Water absorption & $1 \mathrm{~h}, 2 \mathrm{~h}, 4 \mathrm{~h}, 8 \mathrm{~h}, 1 \mathrm{~d}, 2 \mathrm{~d}, 4 \mathrm{~d}, 7 \mathrm{~d}, 14 \mathrm{~d}, 28 \mathrm{~d}, 90 \mathrm{~d}, 180 \mathrm{~d}$ \\
Tension & $7 \mathrm{~d}, 14 \mathrm{~d}, 28 \mathrm{~d}, 90 \mathrm{~d}, 180 \mathrm{~d}$ \\
\hline
\end{tabular}

Note: $\mathrm{h}$ and $\mathrm{d}$ represent hour and day, respectively. 


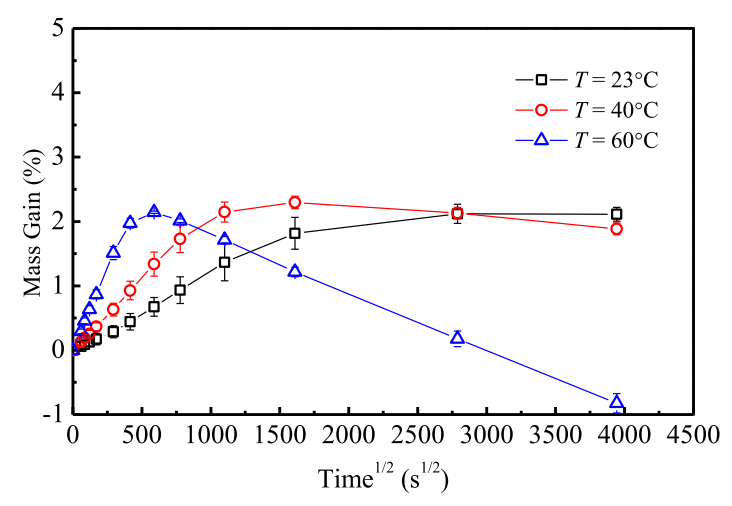

(a)

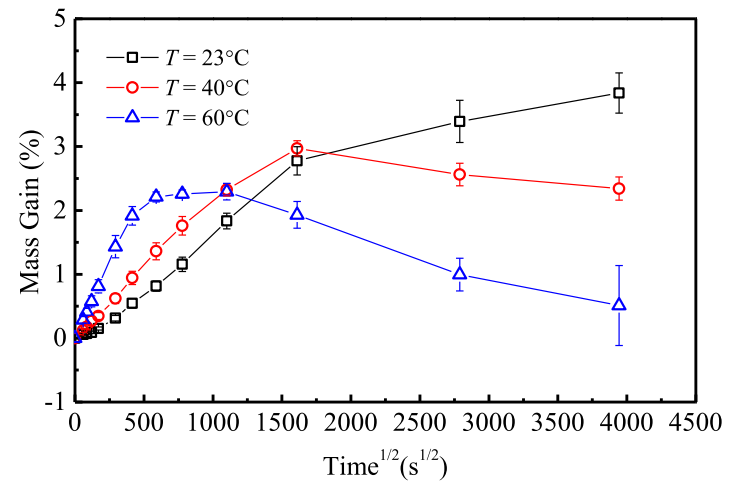

(b)

Fig. 3. Relationship between the mass gain and the aging time for specimens in Group A: (a) immersed in distilled water; (b) immersed in alkaline solution.

i.e., an ascending and then a descending phase respectively. In the ascending branch, the mass gain of the resins increases with the aging time until the peak water absorption is reached. When the test temperatures of the specimens were $23^{\circ} \mathrm{C}, 40^{\circ} \mathrm{C}$ and $60^{\circ} \mathrm{C}$, the peak water absorptions were $2.12 \%, 2.30 \%$ and $2.14 \%$ for the distilled water, and were $3.84 \%, 2.97 \%$ and $2.31 \%$ for the alkaline solution, respectively. It can also be observed that the mass gain of the resins immersed in alkaline solution was generally higher than that immersed in distilled water for the same aging times. After the peak water absorption was reached, the mass gain decreased as the aging time increased. The reason for such phenomenon might be that the resin matrix structure degraded gradually due to capillary cracks caused by the water diffusion. For the specimen immersed in $60^{\circ} \mathrm{C}$ distilled water with the duration of 180 days, as shown in Fig. 3(a), the mean residual mass was less than its initial mass, which indicates that the epoxy resin suffered a severe degradation reaction at this temperature.

Fig. 4 shows the relationship between the mass gain and aging time (in seconds) for the specimens of Group B with different thicknesses which presents that the trend of water uptake also follows a two-stage diffusion response. In the first stage, the thicker specimen had a smaller water uptake when the aging test time was the same. For the specimens with a thickness of $1 \mathrm{~mm}, 2$ $\mathrm{mm}$ and $4 \mathrm{~mm}$, their peak water absorptions were $2.24 \%, 2.14 \%$ and $2.01 \%$ for the distilled water, and $2.35 \%, 2.31 \%$ and $2.26 \%$ for the alkaline solution, respectively. The coefficients of variation of the peak mass gains were 0.05 and 0.02 for distilled water and alkaline solution, respectively, which indicated that the peak mass gains were almost same for the specimens with different thicknesses. This observation was consistent with the findings in the lit- eratures $[32,38]$. This finding was reasonable because that the degradation mechanism, which would affect the peak mass gain, was hardly influenced by the thickness of specimens. After the peak water uptake was reached, the mass gain decreased with the increase of aging time. Moreover, the decrease rate of the mass gain increased with the reduction of the specimen thickness, which indicates that the specimen with a smaller thickness is more likely to be eroded at a given duration. Similarly, this observation can also be explained by assuming that the resin matrix structure was damaged due to the diffusion of water.

\subsection{Tensile test}

For the specimens in Group A, the tested tensile strength retentions are plotted against their aging time (in days) in Fig. 5(a) and (b) for distilled water and alkaline solution, respectively. It can be observed that the tensile strength retention decreased rapidly in the first month with the increase of test duration and then kept relatively a constant. At the test temperatures of $23^{\circ} \mathrm{C}, 40^{\circ} \mathrm{C}$ and 60 ${ }^{\circ} \mathrm{C}$, the tensile strength retentions at 180 days were $83.11 \%, 70.33 \%$, $64.64 \%$ for the distilled water, and were $79.80 \%, 73.31 \%, 58.78 \%$ for the alkaline solution, respectively. Fig. 6 shows the relationship between the tensile strength retention and aging time (in days) for the specimens in Group B. When the specimens had a thickness of $1 \mathrm{~mm}, 2 \mathrm{~mm}$ and $4 \mathrm{~mm}$, the tensile strength retentions at 180 days were $58.72 \%, 64.64 \%, 67.46 \%$ for the distilled water, and $59.08 \%, 58.78 \%, 66.14 \%$ for the alkaline solution, respectively. It can be concluded that the tensile strength decreased more significantly when the test temperature were higher. On the other hand,

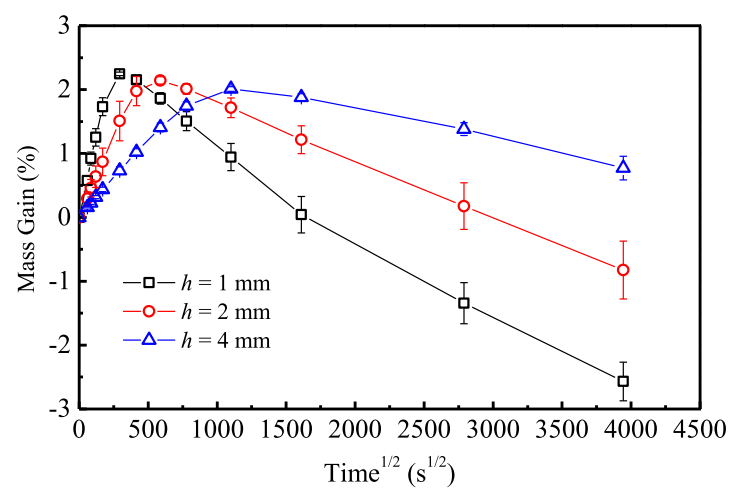

(a)

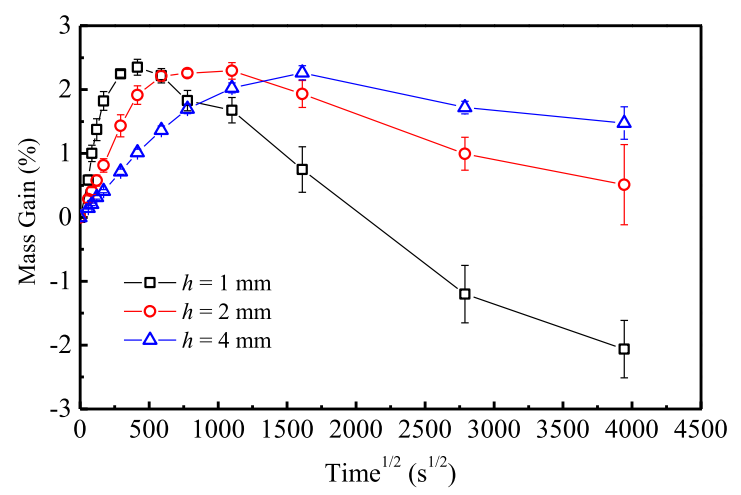

(b)

Fig. 4. Relationship between the mass gain and the aging time for specimens in Group B: (a) immersed in distilled water; (b) immersed in alkaline solution. 


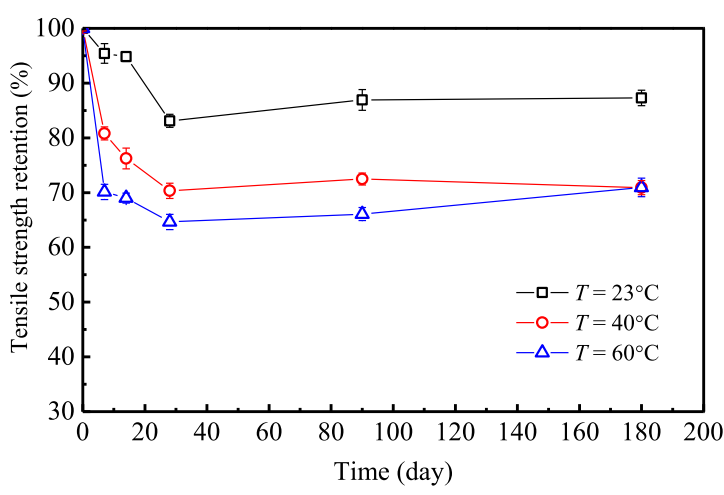

(a)

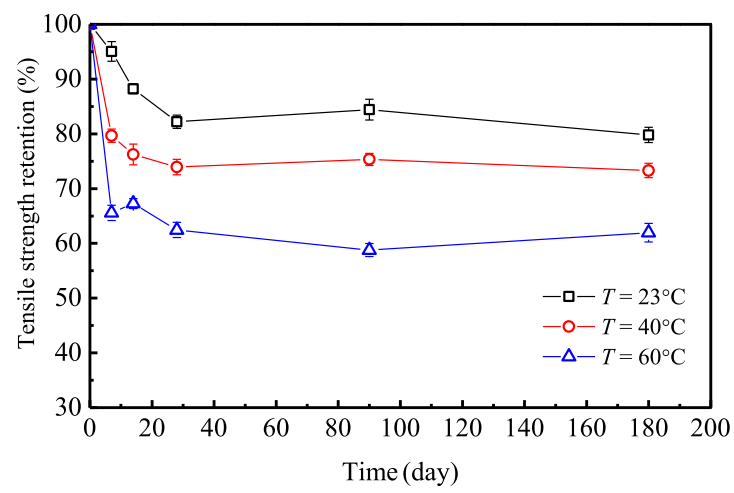

(b)

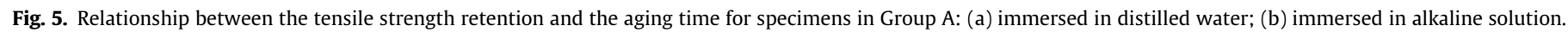

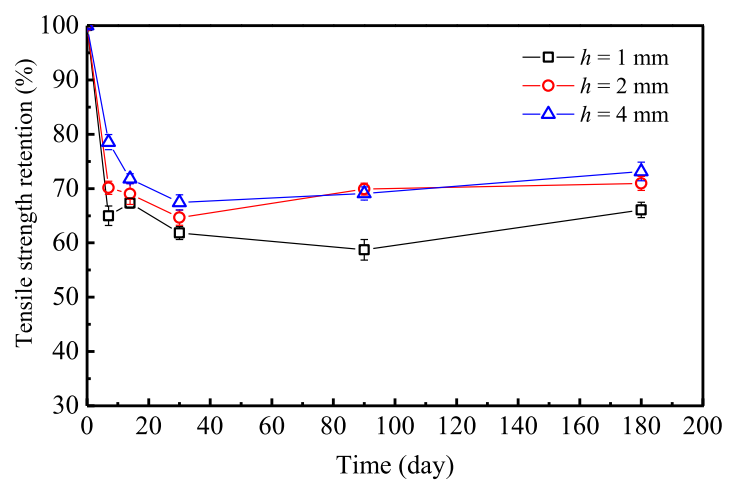

(a)

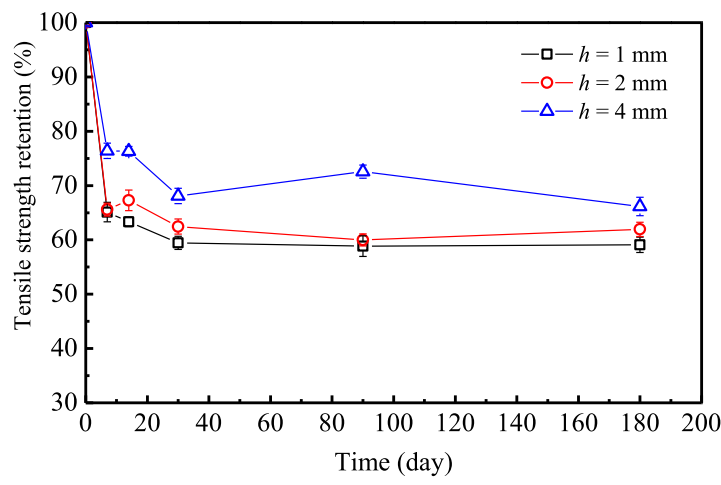

(b)

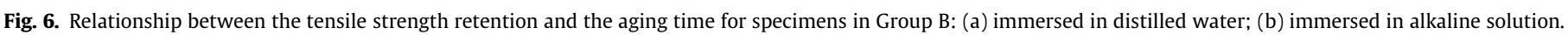

when the temperature was constant, a smaller thickness resulted in a larger degradation in the tensile strength of the resin material.

\subsection{Results of scanning electron microscope (SEM) experiment}

As described previously, Figs. 3 and 4 indicate that the mass gain decreased with the increase of aging time after the quasiequilibrium water absorption of specimens was reached. To explain this phenomenon, the micro-structure of the specimens was investigated by the SEM technology. As shown in Fig. 7, the results show that a lot of capillary cracks and voids were generated in the resin specimen $(h=2 \mathrm{~mm})$ after it was immersed in $60^{\circ} \mathrm{C}$ distilled water or alkaline solution for 180 days. The defects lead to the further decrease of the mass gain in the second diffusion stage.

\section{Discussions on accelerating effect}

In this section, the TAM and ThAM are adopted to process the test data of the specimens in Groups A and B, respectively. The test

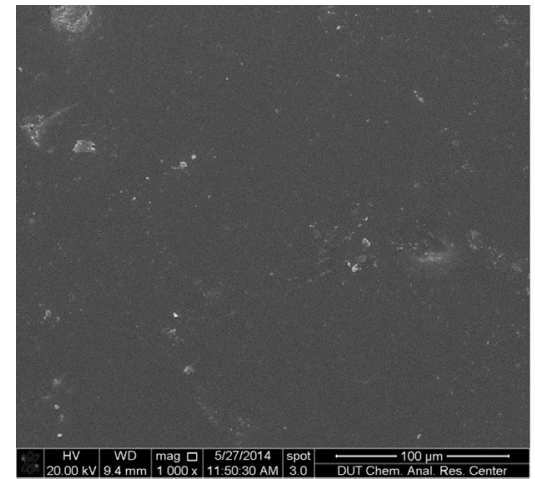

(a)

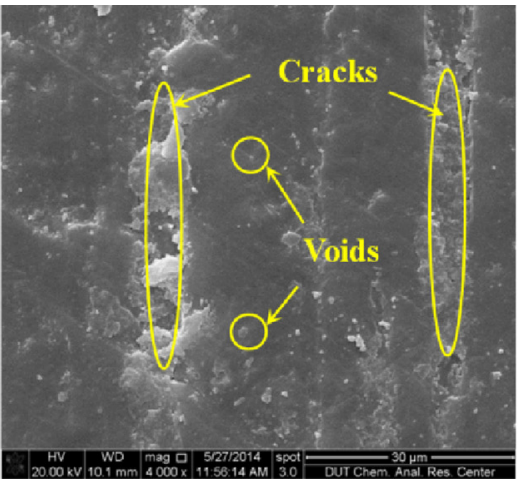

(b)

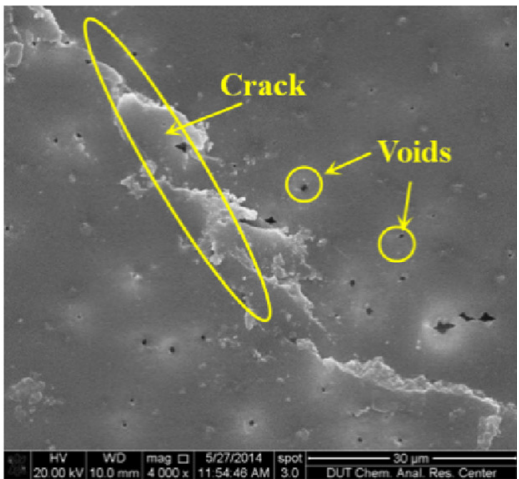

(c)

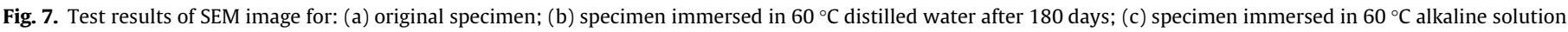
after 180 days. 
results for different temperatures or specimen thicknesses are first converted under a same aging time by the corresponding acceleration factors, and then the trends of the water absorption or tensile strength retention are given by a regression analysis. By comparing the processed test data based on the two methods, the accelerating effects of the aging tests of resin materials are discussed.

\subsection{Accelerating procedure based on TAM}

Eq. (2) indicates that the temperature acceleration factor $A F_{T}$ is not only dependent on the temperature, but is also influenced by the activation energy of the test solutions. Thus, it is crucial to obtain the value of the activation energy prior to calculating the factor $A F_{T}$. As mentioned above, it was assumed that the degradation mechanism of the test material is not changed as test temperature changes, thus Eq. (1) is transformed into Eq. (17). It indicates that the degradation rate is a function of test temperature. In the equation, the activation energy of test solutions is calculated according to [24].

$-\ln (D)=\left(\frac{E_{a}}{R}\right) \frac{1}{T}-\ln (A)$

The degradation rate $D$ can be obtained by regressing the test data at different temperatures. For the water uptake of the nonFickian resin used in the current study, Eq. (18) [32] is used to determine the degradation rate in Eq. (17) with the test results shown in Fig. 3.

$M(t)=M_{m}(1+k \sqrt{t})\left\{1-\exp \left[-7.3\left(\frac{D t}{h^{2}}\right)^{0.75}\right]\right\}$

where $k$ represents the relaxation constant of the resin structure in the second diffusion stage.

For the tensile strength retention $y$, Eq. (19) can be used to determine the degradation rate in Eq. (17) with the data shown in Fig. 6, in which $a$ and $b$ are constants.

$y=a \exp \left(-\frac{t}{D}\right)+b$

The regression coefficients and the correlation coefficients (i.e., $r$ ) of Eqs. (18) and (19) are listed in Tables 3 and 4, which show that the degradation rates are determined for the water uptake and tensile strength retention, respectively. For the water uptake, $-\ln (D)$ is plotted against $1 / T$ in Fig. 8 , which shows that the activation energy $E_{a}$ is determined as $62.23 \mathrm{~kJ} / \mathrm{mol}$ and $58.11 \mathrm{~kJ} / \mathrm{mol}$ for distilled water and alkaline solution, respectively. In Fig. $9, \ln (D)$ is plotted against $1 / T$ for the tensile strength retention of the resin epoxy, from which the activation energy $E_{a}$ is calculated as 36.14 $\mathrm{kJ} / \mathrm{mol}$ and $32.32 \mathrm{~kJ} / \mathrm{mol}$ for distilled water and alkaline solution, respectively.

With the activation energy known, the factor $A F_{T}$ can be calculated with Eq. (2) for the specimens under different temperatures and listed in Table 5. Figs. 3 and 6 are transformed to Figs. 10 and 11 by multiplying aging times at $40^{\circ} \mathrm{C}$ and $60^{\circ} \mathrm{C}$ with corresponding $A F_{T}$ values. It is shown that the water uptake and the tensile strength retention can be predicted up to 8.1 and 2.5 years, respectively. Similarly, based on the processed test data of specimens exposed to $60^{\circ} \mathrm{C}$, the trends of the water absorption and tensile strength retention of the material are given by regression and shown in Figs. 10 and 11. It can be seen that the processed test data of $23^{\circ} \mathrm{C}$ and $40^{\circ} \mathrm{C}$ do not follow the trend of test results of $60^{\circ} \mathrm{C}$. This finding is consistent with Fig. 3. The trend of mass gain at $60{ }^{\circ} \mathrm{C}$, however, was quite different from those at $23^{\circ} \mathrm{C}$ and $40{ }^{\circ} \mathrm{C}$ after the quasi-equilibrium water uptake was reached. This is mainly because of the change of the degradation mechanism due to the elevation of temperature.

\subsection{Accelerating procedure based on ThAM}

A similar procedure was used to process the test data in Group B using the ThAM to accelerate the aging tests of the resin. Since the maximum duration adopted in this study does not exceed the effective duration, the ThAM can be used to accelerate both the tests of water absorption and tensile strength. For the specimens with a thickness of $4 \mathrm{~mm}, 2 \mathrm{~mm}$ and $1 \mathrm{~mm}$, the $A F_{H}$ is calculated as 1,4 , and 16 , respectively, based on Eq. (10). Then the test results of the water absorption and the tensile strength retention (i.e., Figs. 5 and 7) were transformed by multiplying aging times for 2 $\mathrm{mm}$ and $4 \mathrm{~mm}$ with corresponding $A F_{H}$ values as shown in Figs. 12 and 13 , respectively. It should be noted that a duration up to 8 years is covered by test data in the current ThAM for both the water absorption and the tensile strength retention of the resin. Based on the processed test data of the specimens with the thickness of $1 \mathrm{~mm}$, the predictions of the water absorption and the tensile strength retention of the resins are obtained by regression as shown in Figs. 12 and 13, respectively. It is shown that the predictions present an overall great agreement with the processed test data of all specimens with different thicknesses. As shown in Fig. 13(a), however, the tensile strength retentions of the specimens with thickness of $2 \mathrm{~mm}$ and $4 \mathrm{~mm}$ immersed in distilled water showed a discrepancy with the trend after an equivalent

Table 3

Regression results for the water uptake of epoxy resin.

\begin{tabular}{|c|c|c|c|c|c|c|c|c|}
\hline \multirow[t]{2}{*}{ Temperature ${ }^{\circ} \mathrm{C}$} & \multicolumn{4}{|c|}{ Distilled water } & \multicolumn{4}{|c|}{ Alkaline solution } \\
\hline & $M_{m}(\%)$ & $k\left(\times 10^{-4}\right)$ & $D\left(\times 10^{-7}\right)$ & $r$ & $M_{m}(\%)$ & $k\left(\times 10^{-4}\right)$ & $D\left(\times 10^{-7}\right)$ & $r$ \\
\hline 23 & 2.36 & -0.27 & 2.03 & 0.99 & 3.15 & 0.55 & 1.75 & 0.99 \\
\hline 40 & 2.71 & -0.77 & 5.62 & 0.99 & 3.69 & -0.96 & 3.30 & 0.99 \\
\hline 60 & 2.73 & -3.33 & 33.45 & 0.99 & 2.87 & -2.15 & 23.86 & 0.99 \\
\hline
\end{tabular}

Table 4

Regression results for the tensile strength retention of epoxy resin.

\begin{tabular}{|c|c|c|c|c|c|c|c|c|}
\hline \multirow[t]{2}{*}{ Temperature ${ }^{\circ} \mathrm{C}$} & \multicolumn{4}{|c|}{ Distilled water } & \multicolumn{4}{|c|}{ Alkaline solution } \\
\hline & $a$ & $D$ & $b$ & $r$ & $a$ & $D$ & $b$ & $r$ \\
\hline 23 & 14.75 & 14.56 & 86.02 & 0.83 & 19.17 & 14.29 & 81.58 & 0.94 \\
\hline 40 & 28.54 & 6.78 & 71.35 & 0.99 & 25.65 & 4.62 & 74.32 & 0.99 \\
\hline 60 & 32.43 & 2.85 & 67.56 & 0.97 & 37.70 & 3.25 & 62.24 & 0.97 \\
\hline
\end{tabular}




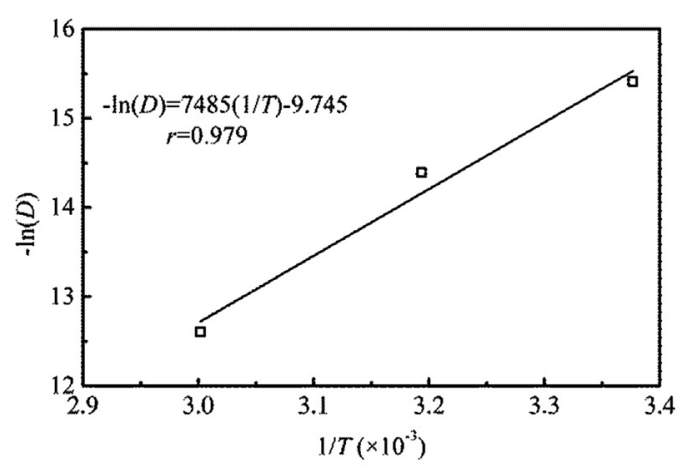

(a)

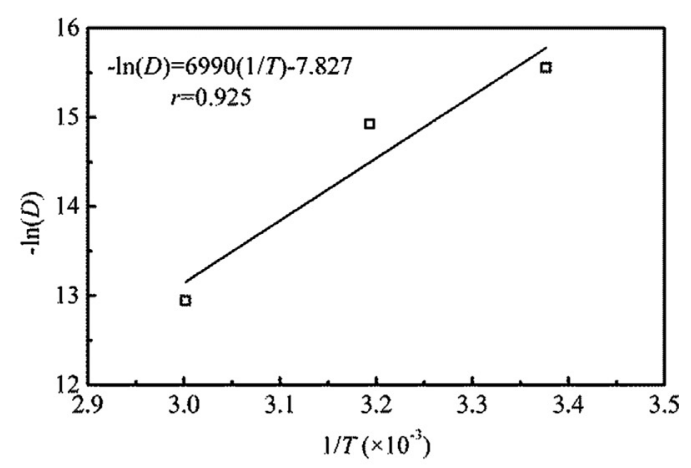

(b)

Fig. 8. Regression results of the activation energy based on the water absorption test: (a) immersed in distilled water; (b) immersed in alkaline solution.

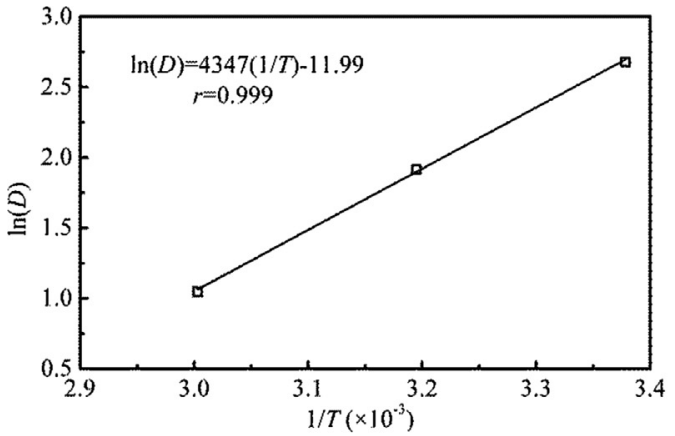

(a)

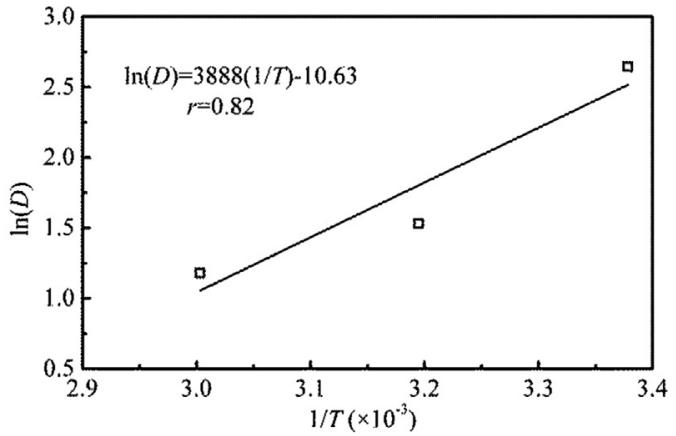

(b)

Fig. 9. Regression results of the activation energy based on the tensile test: (a) immersed in distilled water; (b) immersed in alkaline solution.

Table 5

$A F_{T}$ for specimens in Group A.

\begin{tabular}{llllll}
\hline \multirow{2}{*}{$\begin{array}{l}\text { Temperature } \\
{ }^{\circ} \mathrm{C}\end{array}$} & \multicolumn{2}{l}{ Water absorption test } & & \multicolumn{2}{l}{ Tensile test } \\
\cline { 2 - 3 } \cline { 5 - 6 } \cline { 5 - 6 } & $\begin{array}{l}\text { Distilled } \\
\text { water }\end{array}$ & $\begin{array}{l}\text { Alkaline } \\
\text { solution }\end{array}$ & & $\begin{array}{l}\text { Distilled } \\
\text { water }\end{array}$ & $\begin{array}{l}\text { Alkaline } \\
\text { solution }\end{array}$ \\
\hline 23 & 1 & 1 & 1 & 1 \\
40 & 3.95 & 1.88 & & 2.22 & 2.04 \\
60 & 16.60 & 13.62 & & 5.11 & 4.30 \\
\hline
\end{tabular}

duration of about 300 days and 180 days, respectively. This is mainly caused by the scatter of the test data in the accelerating test.

\subsection{Comparison between TAM and ThAM}

It can be seen from the accelerating procedures that the application of ThAM is much easier than that of TAM. This is mainly due to the fact that the activation energy must be determined when adopting TAM, and thus an additional regression analysis of the test data is inevitable. Since the activation energy is calculated from the test data, as shown in Figs. 8 and 9, the test results corresponding to at least three different temperatures should be provided. This implies that TAM may be inappropriate to be used in the test program with less than three temperatures. Comparatively, the acceleration factor of ThAM is only related to the thickness of specimens. Therefore, it is easily used for wider range. The accelerated efficiencies of TAM and ThAM can be evaluated by

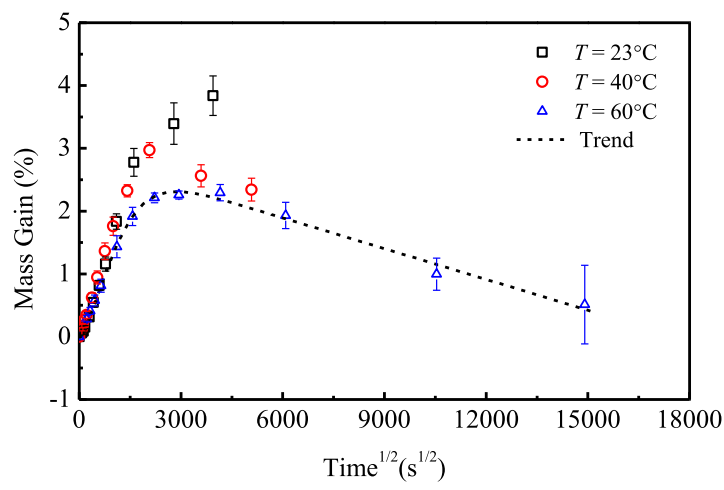

(b)

Fig. 10. Transformed results of water absorption with TAM for specimens in Group A: (a) immersed in distilled water; (b) immersed in alkaline solution. 


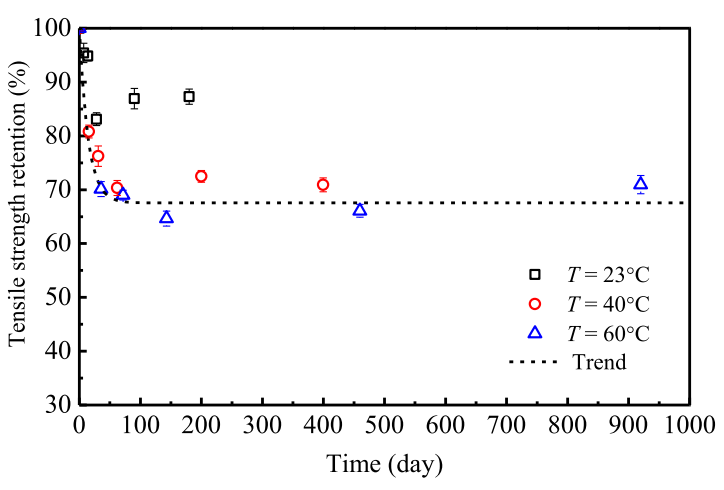

(a)

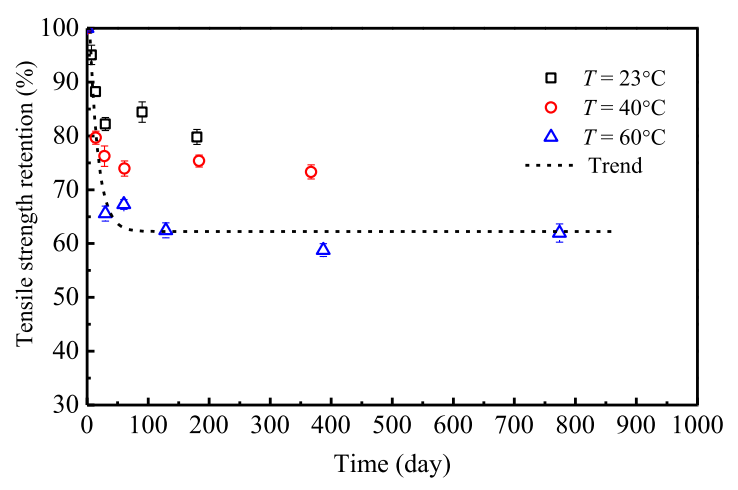

(b)

Fig. 11. Transformed results of tensile strength retention with TAM for specimens in Group A: (a) immersed in distilled water; (b) immersed in alkaline solution.

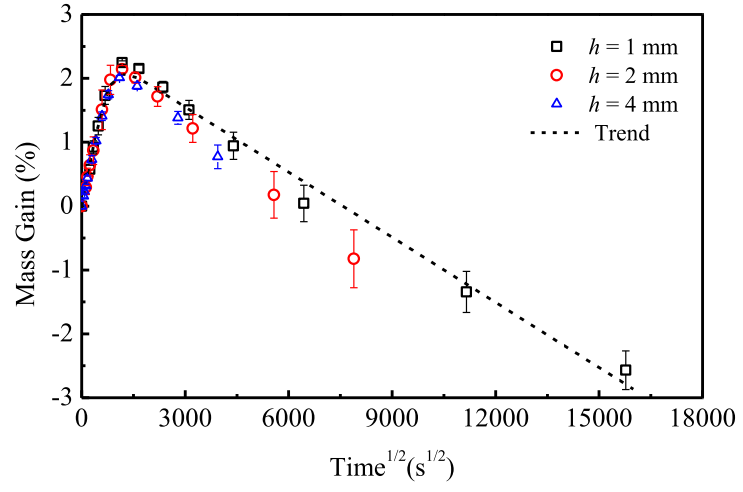

(a)

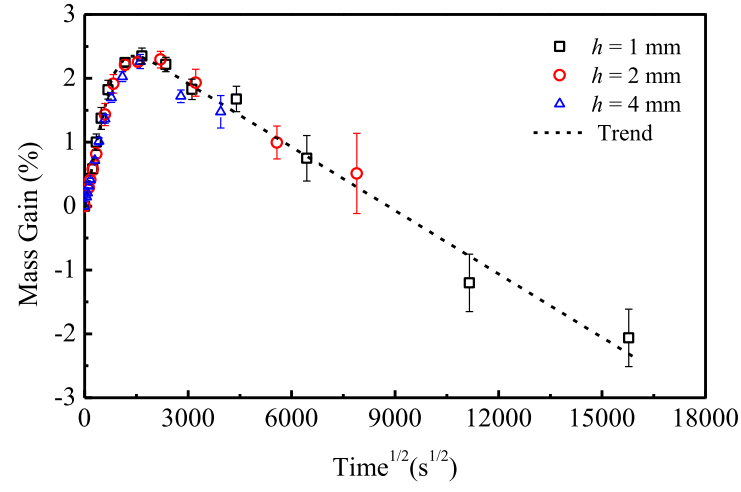

(b)

Fig. 12. Transformed results of water absorption with ThAM for specimens in Group B: (a) immersed in distilled water; (b) immersed in alkaline solution.

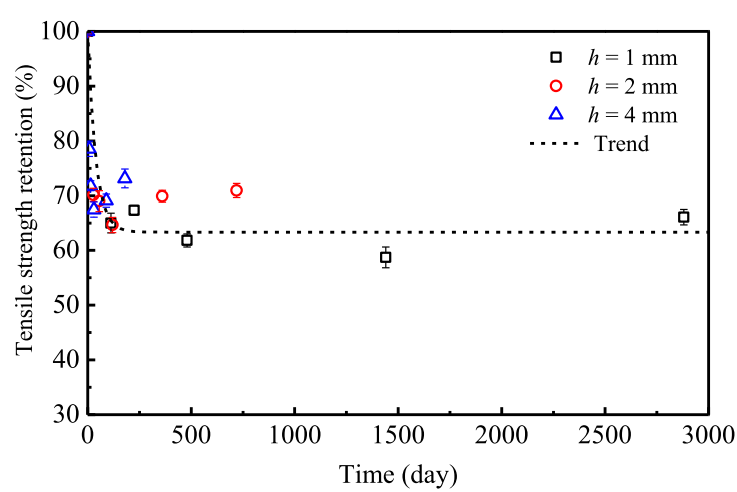

(a)

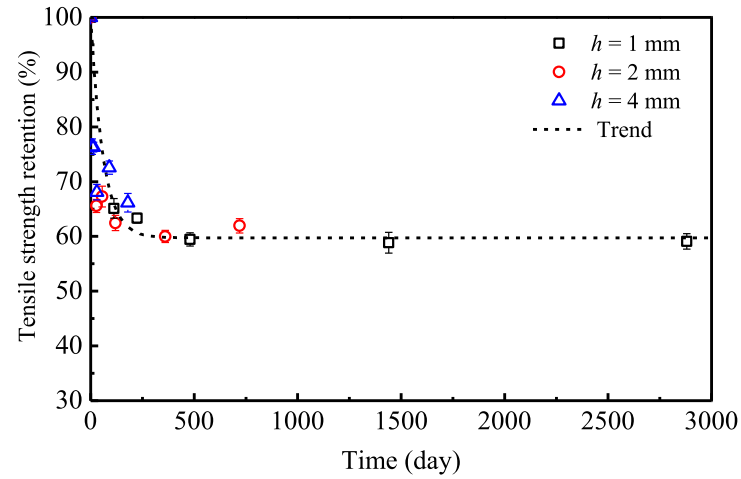

(b)

Fig. 13. Transformed results of tensile strength retention with ThAM for specimens in Group B: (a) immersed in distilled water; (b) immersed in alkaline solution.

comparing the acceleration factors. Eq. (2) shows that the accelerated factor is closely related to the activation energy, which is sensitive to both solution and resin. As shown in Table 5, the maximum $A F_{T}$ obtained based on the activation energies in the current study are 16.60 and 5.11 for water uptake and tensile strength retention, respectively. It should be noted that different accelerated factors will be obtained when different types of resin and/or solutions are adopted. In other words, the accelerated efficiency of TAM is probably limited for certain resin and solution with moderate activation energy. However, such concerns cannot be found for the accelerated efficiency of ThAM. Therefore, when the thick- ness of the specimens is adequately designed, a stable accelerated effect can be ensured.

It can be seen from Fig. 10 that, after processed with TAM, the test data of the specimens with different temperatures show a good consistency in the ascending branch, but then reveal a large discreteness in the descending stage. This is because the structure of resin matrix is destroyed by the solution after a certain duration which probably results in a change of the degradation mechanism. For some types of resin, this degradation may be enhanced at a relative high temperature, which further leads to invalid accelerated results. For example, in the current study, the mass gains of resin 
show significant differences at different temperatures, and TAM is finally verified to be failed to reasonably accelerate the aging process of test materials. In a word, the TAM is limited in some cases, especially when the degradation mechanism of materials may be changed for different temperatures. However, this situation is not observed in Figs. 12 and 13, which supports that the test data processed with ThAM are in good consistency with an identical trend. This is explained by the fact that the temperature remains unchanged and thus the degradation mechanism of resin is scarcely influenced in the test method. The results plotted in Figs. 12 and 13 imply that the predictions show a good agreement with the test data processed with ThAM. This agreement is better than with TAM.

\section{Conclusions}

In this study, a novel accelerated aging method based on the thickness of specimens was preliminarily explored. An experimental program was designed to study water absorption and tensile strength of epoxy resin. The proposed thickness-based accelerated method (ThAM) was then primarily applied to process the test results of short-term properties of the tested materials. Based on the results, the main conclusions can be summarized as follows.

1. The available temperature accelerated method (TAM) presented large discreteness when using high temperature which may change the degradation mechanism of the resin. The acceleration factor of TAM is greatly dependent on the type of solution which further limits the accelerated effect.

2. Compared with TAM, the proposed ThAM is much easier to apply and whose efficiency is scarcely influenced by resin matrix and solution adopted in the test. Moreover, a more stable accelerated effect can be ensured by using ThAM if the thickness of specimens is adequately designed.

3. The proposed ThAM is effective to accelerate aging tests of the epoxy resin by processing the short-term test data with the analysis models. In the future, however, the long-term exposure test results are still needed to verify the proposed models.

\section{Conflict of interest}

None.

\section{Acknowledgments}

The financial supports from the National Key R\&D Program of China with Grant No. 2017YFC0703008, the National Natural Science Foundation of China with Grant Nos. 51508069 and 51778102, the Fundamental Research Funds for the Central Universities with Grant No. DUT17RC(4)17 and DUT18LK35, are greatly acknowledged.

\section{References}

[1] M. Frigione, M. Lettieri, A.M. Mecchi, Environmental effects on epoxy adhesives employed for restoration of historical buildings, J. Mater. Civil Eng. 18 (5SI) (2006) 715-722.

[2] Q. Yu, Y. Wu, Fatigue durability of cracked steel beams retrofitted with highstrength materials, Constr. Build. Mater. 155 (2017) 1188-1197.

[3] D. Zhang, X. Gu, Q. Yu, H. Huang, B. Wan, C. Jiang, Fully probabilistic analysis of FRP-to-concrete bonded joints considering model uncertainty, Compos. Struct. 185 (2018) 786-806.

[4] J. Dai, Y. Bai, J.G. Teng, Behavior and modeling of concrete confined with FRP composites of large deformability, J. Compos. Constr. 15 (6) (2011) 963-973.

[5] T. Mohammadi, B. Wan, K.A. Harries, M.E. Sweriduk, Bond behavior of FRPconcrete in presence of intermediate crack debonding failure, J. Compos. Constr. 21 (5) (2017) 4017018.

[6] Y. Bai, J. Dai, J.G. Teng, Buckling of steel reinforcing bars in FRP-confined RC columns: an experimental study, Constr. Build. Mater. 140 (2017) 403-415.
[7] Y. Pan, G. Xian, M.A.G. Silva, Effects of water immersion on the bond behavior between CFRP plates and concrete substrate, Constr. Build. Mater. 101 (1) (2015) 326-337.

[8] Z. Wang, X. Zhao, G. Xian, G. Wu, R.K.S. Raman, S. Al-Saadi, et al., Long-term durability of basalt- and glass-fibre reinforced polymer (BFRP/GFRP) bars in seawater and sea sand concrete environment, Constr. Build. Mater. 139 (2017) 467-489.

[9] Z. Lu, G. Xian, H. Li, Effects of elevated temperatures on the mechanical properties of basalt fibers and BFRP plates, Constr. Build. Mater. 127 (2016) 1029-1036.

[10] Y. Huang, Y. Guo, Review of durability of Fiber Reinforced Polymer (FRP) reinforced concrete structure, Appl. Mech. Mater. 548-549 (2014) 1651-1654.

[11] J.M. Sousa, J.R. Correia, S. Cabral-Fonseca, Durability of an epoxy adhesive used in civil structural applications, Constr. Build. Mater. 161 (2018) 618-633.

[12] J. Zhou, J.P. Lucas, Hygrothermal effects of epoxy resin. Part I: the nature of water in epoxy, Polymer 40 (20) (1999) 5505-5512.

[13] J. Zhou, J.P. Lucas, Hygrothermal effects of epoxy resin. Part II: variations of glass transition temperature, Polymer 40 (20) (1999) 5513-5522.

[14] P. Nogueira, C. Ramirez, A. Torres, M.J. Abad, J. Cano, J. Lopez, et al., Effect of water sorption on the structure and mechanical properties of an epoxy resin system, J. Appl. Polym. Sci. 80 (1) (2001) 71-80.

[15] F. Lapique, K. Redford, Curing effects on viscosity and mechanical properties of a commercial epoxy resin adhesive, Int. J. Adhes. Adhes. 22 (2002) 337-346.

[16] Y.C. Lin, X. Chen, Moisture sorption-desorption-resorption characteristics and its effect on the mechanical behavior of the epoxy system, Polymer 46 (25) (2005) 11994-12003.

[17] L. Goglio, M. Rezaei, Variations in mechanical properties of an epoxy adhesive on exposure to warm moisture, J. Adhes. Sci. Technol. 28 (2014) 1394-1404.

[18] P. Silva, P. Fernandes, J. Sena-Cruz, J. Xavier, F. Castro, D. Soares, et al., Effects of different environmental conditions on the mechanical characteristics of a structural epoxy, Compos. Part B-Eng. 88 (2016) 55-63.

[19] M. Savvilotidou, A.P. Vassilopoulos, M. Frigione, T. Keller, Development of physical and mechanical properties of a cold-curing structural adhesive in a wet bridge environment, Constr. Build. Mater. 144 (2017) 115-124.

[20] Y. Wang, Y. Wang, B. Wan, B. Han, G. Cai, Z. Li, Properties and mechanisms of self-sensing carbon nanofibers/epoxy composites for structural health monitoring, Compos. Struct. 200 (2018) 669-678.

[21] Y. Wang, G. Chen, B. Wan, H. Lin, J. Zhang, Behavior of innovative circular ice filled steel tubular stub columns under axial compression, Constr. Build. Mater. 171 (2018) 680-689.

[22] Y. Miyano, M. Nakada, N. Sekine, Accelerated testing for long-term durability of FRP laminates for marine use, J. Compos. Mater. 39 (1) (2005) 5-20.

[23] Y. Chen, J.F. Davalos, I. Ray, Durability prediction for GFRP reinforcing bars using short-term data of accelerated aging tests, J. Compos. Constr. 10 (4) (2006) 279-286.

[24] Y. Chen, J.F. Davalos, I. Ray, H. Kim, Accelerated aging tests for evaluations of durability performance of FRP reinforcing bars for concrete structures, Compos. Struct. 78 (1) (2007) 101-111.

[25] Y. Miyano, M. Nakada, Life Prediction of CFRP Laminates based on Accelerated Testing Methodology, Conference Proceedings of the Society for Experimental Mechanics Series, 2017, pp. 35-47.

[26] Z. Wang, X. Zhao, G. Xian, G. Wu, R.K.S. Raman, S. Al-Saadi, Effect of sustained load and seawater and sea sand concrete environment on durability of basaltand glass-fibre reinforced polymer (B/GFRP) bars, Corros. Sci. 138 (2018) 200218.

[27] K.J. Laidler, The development of the Arrhenius equation, J. Chem. Educ. 61 (6) (1984) 494-498.

[28] M. Robert, P. Wang, P. Cousin, B. Benmokrane, Temperature as an accelerating factor for long-term durability testing of FRPs: should there be any limitations?, J Compos. Constr. 14 (4) (2010) 361-367.

[29] L.R. Bao, A.F. Yee, Effect of temperature on moisture absorption in a bismaleimide resin and its carbon fiber composites, Polymer 43 (2002) 3987-3997.

[30] B. Benmokrane, A. Manalo, J. Bouhet, K. Mohamed, M. Robert, Effects of diameter on the durability of glass fiber-reinforced polymer bars conditioned in alkaline solution, J. Compos. Constr. 21 (5) (2017) 40170405.

[31] K.D. Dorkenoo, P.H. Pfromm, Experimental evidence and theoretical analysis of physical aging in thin and thick amorphous glassy polymer films, J. Polym. Sci. Part B: Polym. Phys. 37 (16) (1999) 2239-2251.

[32] L.R. Bao, A.F. Yee, C. Lee, Moisture absorption and hygrothermal aging in a bismaleimide resin, Polym. 42 (17) (2001) 7327-7333.

[33] K. Tsuda, Behavior and mechanisms of degradation of thermosetting plastics in liquid environments, J. Jpn. Petrol. Inst. 50 (5) (2007) 240-248.

[34] H. Hojo, K. Tsuda, K. Ogasawara, Form and rate of corrosion of corrosionresistant FRP resins, Adv. Compos. Mater. 1 (1) (1991) 55-67.

[35] ACI, 440.3R-04, Guide test methods for fiber-reinforced polymers(FRPs) for reinforcing or strengthening concrete structures, ACI Committee (2004).

[36] ASTM D570-98(2010)e1, Standard Test Method for Water Absorption of Plastics, ASTM International, 2010.

[37] ASTM D638-14, Standard test Method for Tensile Properties of Plastics, ASTM International, 2014.

[38] Y.C. Lin, X. Chen, Moisture sorption-desorption-resorption characteristics and its effect on the mechanical behavior of the epoxy system, Polym. 46 (25) (2005) 11994-12003. 